\title{
Bovine respiratory disease (BRD) cause-specific and overall mortality in preweaned calves on California dairies: The BRD 10K study
}

\author{
S. A. Dubrovsky, ${ }^{1}$ A. L. Van Eenennaam, ${ }^{1}$ B. M. Karle, ${ }^{2}$ P. V. Rossitto, ${ }^{3}$ T. W. Lehenbauer,${ }^{3,4}$ and S. S. Aly ${ }^{3,4 *}$ \\ ${ }^{1}$ Department of Animal Science, University of California, Davis 95616 \\ ${ }^{2}$ Cooperative Extension, Division of Agriculture and Natural Resources, University of California, Orland 95963 \\ ${ }^{3}$ Veterinary Medicine Teaching and Research Center, School of Veterinary Medicine, University of California, Davis, Tulare 93274 \\ ${ }^{4}$ Department of Population Health and Reproduction, School of Veterinary Medicine, University of California, Davis 95616
}

\section{ABSTRACT}

Mortality in preweaned dairy calves is a significant source of economic loss for dairy producers. In particular, bovine respiratory disease (BRD) is a leading cause of death in preweaned dairy calves. The objectives of this study were to investigate management practices and their effects on mortality, both that specifically attributed to BRD and overall mortality due to all causes, in preweaned dairy calves. Rates of failure of passive transfer of immunity (FPT) are also reported. The study consisted of a convenience sample of 5 dairies across California, selected based on management practices, calf records, location, and size. Trained study personnel performed comprehensive calf management surveys on every dairy at least once every season. Calves were enrolled in the study at birth and followed until weaning. Mixed-effect logistic regression models were specified for the outcomes all-cause mortality (any death before weaning) and mortality attributed to BRD. The 2 final models included a total of 11,470 calves that were born on the study dairies and followed until weaning. The study cohort's overall crude mortality was $2.8 \%$, with crude mortality of individual dairies ranging from 1.7 to $7.2 \%$. The proportion of mortality attributed to BRD was $19.3 \%$, with a range of 0 to $27.1 \%$ on the study dairies. Increasing the frequency of changing maternity pen bedding was associated with a decreased risk of mortality due to BRD. Calves diagnosed with BRD in the spring had an increased risk of mortality compared with calves born in the summer; mortality in calves with fall and winter BRD diagnoses did not different significantly from that in summer. Season of mortality was not significant in either model. Feeding $\geq 5.7 \mathrm{~L}$ of milk per day per calf (vs. $\leq 3.7 \mathrm{~L} / \mathrm{d}$ ) decreased the risk of mortality in calves over $21 \mathrm{~d}$ of

Received July 28, 2018.

Accepted April 9, 2019.

*Corresponding author: saly@ucdavis.edu age. Twins had a $68 \%$ increased risk of all-cause mortality compared with calves born as singletons. Both mortality models showed an association between administration of a modified live vaccine in dams (targeting BRD pathogens) and a decreased risk of mortality in calves. Using a serum total protein cut-off of $5.2 \mathrm{~g} /$ $\mathrm{dL}, 16.8 \%$ of calves had FPT, with a mean serum total protein concentration of $5.94 \pm 0.06 \mathrm{~g} / \mathrm{dL}$ across all calves sampled.

Key words: bovine respiratory disease, epidemiology, mortality

\section{INTRODUCTION}

Preweaning (postnatal) calf mortality in dairy calves in the United States represents an ongoing challenge for dairy producers but has the potential to be reduced through the use of management practices that prevent diseases and environmental conditions that increase mortality risk (Tozer and Heinrichs, 2001). The most recent studies have reported postnatal mortality rates in preweaned calves of $5.0 \%$ (calves were enrolled at $24 \mathrm{~h}$ of age) in the most recent USDA National Animal Health Monitoring Systems (NAHMS) Dairy 2014 study, whereas a study done in northern Mexico examining postnatal (excluding calves from difficult calvings) to $21 \mathrm{~d}$ of age had a range of 7.0 to $29.1 \%$ and an overall mortality of $14 \%$ (Mellado et al., 2014; Urie et al., 2018). Windeyer et al. (2014) reported that diarrhea and bovine respiratory disease (BRD) are the 2 primary causes of preweaned dairy calf mortality. Sivula et al. (1996) reported that mortality between birth and 10 wk of age was $7.6 \%$, with $29.7 \%$ of deaths as a result of pneumonia, during the 16-mo study period on 30 Holstein herds in Minnesota. Those authors further reported a case fatality rate of $9.2 \%$ for calves diagnosed with BRD. A nationwide study of dairy calf morbidity and mortality as part of the calf component of the 2014 NAHMS reported that BRD was the identified cause for $14.1 \%$ of deaths, with a case fatality rate of $6.0 \%$ (Urie et al., 2018). These numbers indicate that there 
is still room for improvement on decreasing overall calf mortality and that significant gains could be made in decreasing mortality by focusing on decreasing the case fatality rate for BRD.

Failure of passive transfer (FPT) is one of the main objective measurements considered when examining dairy calf management. Beam et al. (2009) reported the prevalence of FPT in US dairy calves to be $19.2 \%$, with $40.7 \%$ of the 413 herds studied having at least one calf with FPT. In a follow-up nationwide study, Shivley et al. (2018) described FPT to be $12.1 \%$ in 1,623 Holstein calves on 101 dairies. A study performed on California dairies in 2014 estimated a $32.6 \%$ FPT proportion using a $5.2 \mathrm{~g} / \mathrm{dL}$ cut-off for serum total protein (TP) concentration (Love et al., 2014). Failure of passive transfer has been shown repeatedly to increase the risk of mortality, and a recent study found that the risk of mortality was 11 times greater in calves with FPT than in calves with adequate passive transfer of immunity (Lora et al., 2018). Urie et al. (2018) reported that an increase in serum IgG levels reduced the risk of mortality in preweaned dairy calves and suggested that IgG concentrations of $15.0 \mathrm{~g} / \mathrm{L}$ would be a more appropriate cutoff to reduce morbidity and mortality. Results of studies that examine the effects of FPT on the development of BRD in preweaned calves are mixed. Virtala et al. (1999) found low IgG levels to be a significant risk factor for the development of pneumonia in preweaned calves as diagnosed by a clinician but not for BRD diagnosed by a calf caregiver. Mahendran et al. (2017) did not find an association between IgG levels and BRD diagnosed utilizing a modified version of the University of Wisconsin calf health scoring system (McGuirk and Peek, 2014).

Other farm management practices, including a longer duration of stay for the newborn calf in the calving pen, housing in groups, and various milk management practices, have been associated with increased preweaning calf mortality (Losinger and Heinrichs, 1997; Gulliksen et al., 2009). Although many studies have described mortality in environments that have harsh winters that require indoor housing of calves and other management factors (Sivula et al., 1996; Lago et al., 2006), few studies have examined calf mortality in arid environments with mild winters. This presents a challenge in finding studies that can be compared with management of calves in California. A recent study conducted in northern Mexico, which has a similar environment to California, found that birth type (singleton or twin), type of milk fed (milk vs. milk replacer), and winter season had a significant effect on risk of mortality (Mellado et al., 2014). The Mexico study focused on seasonal variables, with milk type fed being the only calf management variable included in their model. Other management practices (e.g., colostrum management and vaccination practices) were not tested in their model. The last study to examine preweaned calf mortality and season and management practices in California was conducted over 40 yr ago (Martin et al., 1975), and calf care and management practices have evolved greatly in the interim. The objective of this prospective cohort study was to identify management factors associated with the risk of crude mortality and risk of mortality caused by BRD in California dairy calves. Specifically, our study sought to examine associations between mortality and dam and calf management practices that could be modified to aid in prevention of calf mortality.

\section{MATERIALS AND METHODS}

The study was approved by the Institutional Animal Use and Care Committee at the University of California, Davis (Protocol \#17496).

\section{Study Herd Selection}

The study herds were selected based on size, location, diversity of management practices, record-keeping practices (whether they kept organized records for calves), and the owner's willingness to participate. Five herds were enrolled in the study between March and August 2015. The study herds (Figure 1) were located in Northern California (2 dairies), the northern San Joaquin Valley (3 dairies), and greater Southern California (1 dairy), specifically chosen to represent the distinct dairy management systems in California based on several characteristics described in Dubrovsky et al. (2019). All dairies selected had participated in previous research through the University of California and kept detailed calf records. The current report summarizes findings related to BRD-specific mortality; findings related to BRD morbidity are presented in a companion article (Dubrovsky et al., 2019).

\section{Calf Enrollment and Exclusion Criteria}

At commencement of the study, all preweaned calves on each dairy were enrolled. Subsequently, calves were enrolled at birth and followed until weaning. Calves that were sold or died before weaning were assigned the date of those events as their exit date. Male calves born on the dairy and sold within $2 \mathrm{~d}$ of birth were not enrolled in the study. Any calves, male or female, sold more than $2 \mathrm{~d}$ after birth were included in the study. Any calves that died within $24 \mathrm{~h}$ of birth or were stillborn were not enrolled in the study. 


\section{Data Collection}

Dairy staff involved in care of enrolled calves were trained by study personnel to identify and record clinical signs of BRD and calf mortality due to BRD. Treatment cards were used to collect calf health information, as described in Dubrovsky et al. (2019). Briefly, treatment cards included spaces for information on calf ID, breed, date of birth, sex, date of treatment, medications administered, and a checklist of clinical signs. If a calf died before weaning, calf managers were instructed to note the date of death on the treatment card and return the cards for dead calves to the study personnel. Dairies' mortality records (computer records and paper records depending on the dairy) were collected and the cause of death from the dairy records was used to define whether a calf's death was attributable to BRD. If dairy records did not provide a cause of death, then calves were defined as having death attributable to BRD if they had been treated for BRD within 30 $\mathrm{d}$ of their death date. All other deaths were recorded and noted as deaths not attributed to BRD for data

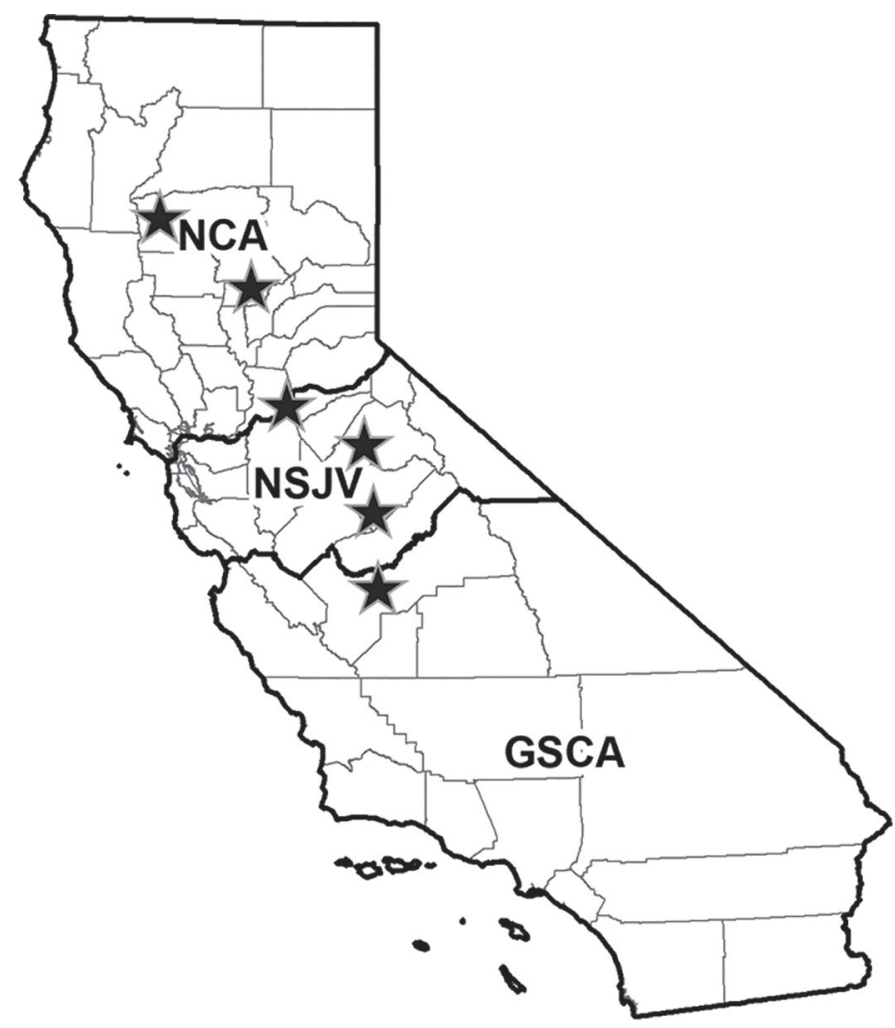

Figure 1. California map identifying Northern California (NCA), northern San Joaquin Valley (NSJV), and greater Southern California (GSCA), which includes the southern half of the San Joaquin Valley. Also identified in the map (stars) are the counties in which dairies were enrolled in a longitudinal study of bovine respiratory disease in preweaned dairy calves. analysis purposes. A comprehensive management survey was administered at enrollment and again every 2 to $4 \mathrm{mo}$ for the duration of the study to incorporate seasonal management changes. At each assessment, trained personnel interviewed the dairy calf manager or owner and asked questions about herd demographics, calving practices, calf housing, colostrum management and feeding, feeding programs for calves, respiratory disease vaccinations, and dust management practices. Questions in the first risk assessment survey referred to the previous 3 mo on the study dairy. Subsequent assessments referred to the period since the last risk assessment.

In addition to the risk assessment, study personnel visited the study herds every 2 to 3 wk for the duration of the study to provide outreach and education to the dairy staff, observe calf feedings and treatments, and observe disease recordings. In addition, during visits, investigators observed management practices and reconciled the observed practices with questionnaire responses and recorded hutch type for newborn calves on dairies with multiple hutch types. Data collected included twinning, assisted reproductive techniques, season, maternity pen management (number of times maternity pen bedding was changed per month), calf housing practices (additional shade structures, housing type, whether calves in adjacent hutches could contact each other, hutch elevation, use of a flush system), calf vaccination (intranasal vaccine or modified live injectable vaccine before weaning), and dam vaccination (use of a killed or modified live vaccine). Data on environment were also collected, including dust in the calf area, whether roads around the calving area were dirt, dust around the calf hutches, and whether dust was observed by the manager in the calf raising area. Data were recorded on colostrum management (pooling of colostrum, testing of immunoglobulin levels, use of colostrum replacer, heat treatment, use of preservative, hours that colostrum was stored before feeding, storage temperature, colostrum from first-calf heifers, evaluation of calves' serum TP as an indicator of FPT, volume of colostrum fed in first $12 \mathrm{~h}$, and colostrum feeding method), milk feeding (feeding order and health status, type of milk fed, feeding of waste milk and milk replacer, pasteurization, testing of bacterial content, volume fed, addition of antibiotics to milk, use of bottle or bucket, reduction of feeding at weaning, and interval between feedings), and the availability of water to the calves.

\section{Data Housing and Relational Database}

The study data were stored in a relational database (Access, Microsoft Corp., Redmond, WA). Dairy sur- 
vey responses were linked to their respective individual calf records (calf ID, birthdate, sex, event of interest, twinning status). Specifically, survey responses were time matched to calf records using calf birthdate and the time interval that the survey covered. Calf IDs were linked to events of interest (date of death, weaning date, BRD diagnosis date, and date sold).

\section{Serum Samples}

At each herd assessment between May and June 2016, serum TP was measured to assess passive transfer of immunity in each of the study herds from a random sample of calves identified by calf ID and a list of random numbers generated using Excel (Microsoft Corp.). The sample size was estimated assuming a $25 \%$ prevalence of FPT and an error bound of 1\%. Blood samples were collected via jugular venipuncture, using an 18-gauge needle into whole-blood collection tubes, from calves that were over $24 \mathrm{~h}$ old and less than $3 \mathrm{~d}$ of age. Blood was collected from all calves if fewer than 6 calves met the sampling criteria. The samples were refrigerated within $2 \mathrm{~h}$ of collection and tested within $2 \mathrm{~d}$ for serum TP. The blood was centrifuged for 10 min at approximately $1,000 \times g$ before approximately $0.05 \mathrm{~mL}$ of serum was pipetted onto a temperaturecompensating refractometer (model no. 300027, SPER Scientific, Scottsdale, AZ) and serum TP was processed and measured by the same study author on all dairies throughout the study. Calves with serum TP values less than the cut-off value of $5.2 \mathrm{~g} / \mathrm{dL}$ were determined to have FPT (Calloway et al., 2002)

\section{Mortality}

Percentages of crude mortality and BRD-specific mortality were calculated as well as the BRD case fatality and the BRD proportional mortality. The BRD case fatality was calculated by dividing the number of calves that died after being diagnosed with BRD by the total number of calves diagnosed with BRD. The BRD proportional mortality was the total number of calf deaths attributed to BRD divided by the total number of calf deaths. Mortality was attributed to BRD if a calf had been treated for BRD within $30 \mathrm{~d}$ of death or dairy records directly indicated BRD as cause of death. A BRD diagnosis was based on calf caretaker recording of clinical signs in treatment records of study calves. Crude mortality was calculated as the total number of dead calves (including any calf mortality attributed to $\mathrm{BRD}$ ) divided by the total number of calves enrolled in the cohort.

\section{Logistic Regression}

Preweaned calves on the participating dairies at the beginning of the study had the study start date as their enrollment date. Calves born after the study began had their birth dates as their enrollment dates. Each calf in the study was followed from its enrollment date to an occurrence of the event of interest or censoring. The 2 events of interest (outcomes) used for survival analysis were death attributed to BRD and all-cause mortality (not differentiating between causes of death). Two separate mixed-effects logistic regression models for mortality were specified. Explanatory variables were first examined using univariate models before being included in the full multivariate model. Censoring otherwise occurred if a calf was weaned or sold. Calf ID was recorded in their hutches during visits to the study herds because certain study dairies had more than one hutch type, and any empty hutches were noted and checked against dairy records for dead or sold calves. Mortality data were analyzed for preweaning deaths only. A manual forward model-building algorithm was applied, entering variables related to the herd and calf demographics, followed by those related to housing, colostrum management, feeding, vaccinations, and dustrelated variables. A full multivariable mixed-effects logistic regression model was specified and included a random effect for dairy to account for lack of independence of data within dairies. All statistical comparisons were based on a $5 \%$ level of significance and performed using a commercial statistical software package (Stata IC 14.2, Stata Corp., College Station, TX). All management variables tested were derived from the list of practices under the data collection section. A more detailed list of variables tested in the mortality models can be found in Dubrovsky et al. (2019). Specific interactions tested included 2-way interaction terms between breed and dairy, between milk replacer use and type of milk fed (saleable vs. waste), and between type of milk fed and whether the milk was pasteurized. Twoway interaction terms between supplementation with colostrum replacer and source of colostrum were tested. The interaction between vaccines administered to cows and the use of colostrum replacer was also tested in the model. Use of both killed and modified live vaccines in pregnant dams was tested for interaction with the volume of colostrum fed in the first $12 \mathrm{~h}$ after birth.

\section{RESULTS}

A total of 11,945 calves, $68.5 \%$ female and $31.5 \%$ male, from the 5 dairies were enrolled between March 2015 and July 2016. More details on the study herds 
Table 1. Failure of passive transfer of immunity (FPT) for 184 calves enrolled in a longitudinal study on the epidemiology characteristics of bovine respiratory disease (BRD) in calves on 5 California dairies from birth to weaning ${ }^{1}$

\begin{tabular}{lcccc}
\hline Dairy & $\begin{array}{c}\text { No. of calves } \\
\text { tested }\end{array}$ & $\begin{array}{c}\text { No. of calves } \\
\text { with FPT }\end{array}$ & $\begin{array}{c}\text { FPT } \\
(\%)\end{array}$ & $\begin{array}{c}\text { Mean (SE) } \\
\text { serum TP (g/dL) }\end{array}$ \\
\hline 1 & 57 & 16 & 28 & $5.5(0.07)$ \\
2 & 18 & 1 & 5.6 & $6.22(0.12)$ \\
3 & 31 & 1 & 3.2 & $6.42(0.12)$ \\
4 & 23 & 9 & 39 & $5.74(\mathrm{~S} 0.22)$ \\
5 & 55 & 4 & 7.2 & $6.18(0.10)$ \\
Total & 184 & 31 & 16.8 & $5.94(0.06)$ \\
\hline
\end{tabular}

${ }^{1}$ The cut-off used for defining FPT was a serum total protein (TP) concentration of less than $5.2 \mathrm{~g} / \mathrm{dL}$.

and their management practices can be found in $\mathrm{Du}$ brovsky et al. (2019). Out of the 11,470 calves used in the model, the average age of enrollment was $3.35 \mathrm{~d}$, with 10,164 of the calves enrolled at birth. Changes in vaccination protocols during the course of the study period are summarized in Dubrovsky et al. (2019). Briefly, approximately 63.5 and $69.9 \%$ of the study calves were from dairies that implemented changes during the study in their dams' modified live or killed vaccine protocols, respectively.

\section{Failure of Passive Transfer of Immunity}

Blood samples from 184 calves were collected on the 5 study dairies. The mean serum TP for all samples collected was $5.94 \mathrm{~g} / \mathrm{dL}$, with a range of 4.3 to $8.7 \mathrm{~g} /$ $\mathrm{dL}$ (SE: 0.06). Table 1 presents the serum TP means by dairy as well as FPT rates. In total, 21 male calves were sampled for FPT with a mean serum TP of 5.41 $\mathrm{g} / \mathrm{dL}(\mathrm{SE}=0.11)$ and $28.6 \% \mathrm{FPT} ; 159$ female calves were sampled for FPT with a mean serum TP of 6.01 $\mathrm{g} / \mathrm{dL}(\mathrm{SE}=0.07)$ and $15.7 \% \mathrm{FPT}$.

\section{Mortality and Logistic Regression}

Of all the calves enrolled in the study cohort, $2.8 \%$ of calves died before weaning $(322 / 11,470)$ and the mean herd-level mortality was $4.1 \%$. Table 2 describes the mortality rates, including BRD-specific mortality and
BRD case fatality rates. The mean age at time of death was 33 d (95\% CI: 5 to 130 d). Results for the 2 multivariate models examining BRD-specific mortality and all-cause mortality are presented in Table 3. For the BRD-specific mortality model, the significant variables included modified live vaccination of dam, season, and frequency of changing of maternity bedding. For the model examining all-cause mortality, significant variables were modified live vaccination of dam, milk fed to calves over $21 \mathrm{~d}$ of age, and whether a calf was born a singleton or twin.

\section{DISCUSSION}

Mortality rates were comparable to those found in previous studies, with an overall preweaned mortality of $2.8 \%$, and a $2.3 \%$ BRD case fatality rate (Johnson et al., 2017; Urie et al., 2018). The current study estimates of mortality were slightly lower than results from recent studies that reported mortality rates ranging from 3.5 to $12.7 \%$ in preweaned calves, with the most recent NAHMS (Urie et al., 2018) reporting a 5.0\% mortality rate for preweaned calves (Buczinski et al., 2014; Windeyer et al., 2014; Chigerwe et al., 2015; Johnson et al., 2017; Mahendran et al., 2017; Urie et al., 2018). The overall percentage of BRD-caused mortality was $19.3 \%$ in the current study, which was similar to $19 \%$ reported by Chigerwe et al. (2015) and $20.4 \%$ by Schaffer et al. (2016) but higher than the $14.1 \%$ mortality caused by

Table 2. Mortality statistics calculated from a cohort of 11,470 calves enrolled in a longitudinal study on the epidemiology characteristics of bovine respiratory disease $(\mathrm{BRD})$ in calves on 5 California dairies from birth to weaning ${ }^{1}$

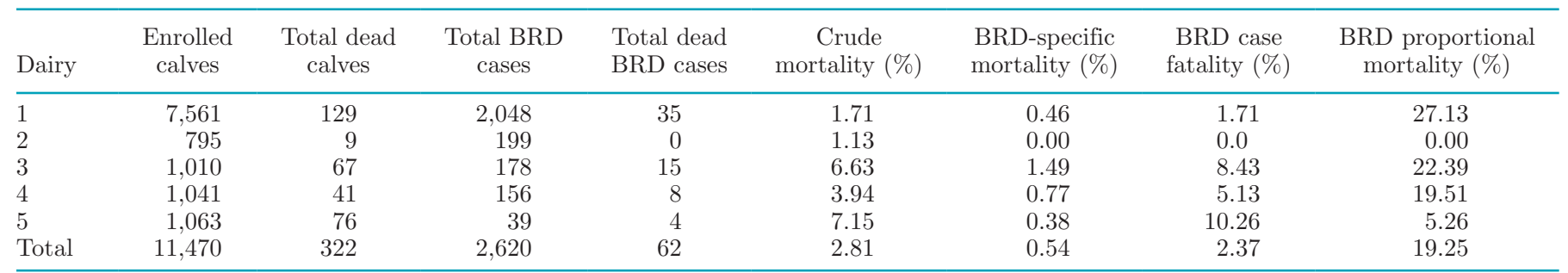

${ }^{1}$ Mortality was attributed to BRD if a calf had been treated for BRD within $30 \mathrm{~d}$ of death or dairy records directly indicated BRD as cause of death. 
respiratory disease in US dairy calves reported by Urie et al. (2018). These rates suggest that the mortality and BRD-specific mortality of the calves in this study are similar to those of US dairy calves as none of the rates reported in this study varied significantly from other recent studies.

A limitation of this study was its partial reliance on dairy records for both the date of death and the cause of death (other than BRD). Although calf caretakers recorded date of death for calves treated for BRD directly on treatment cards, any deaths due to other causes were taken directly from the dairies' records. This is common practice for large studies on calf mortality; however, for future studies, it would be preferable for study personnel to examine the dead calves and take a history or for necropsies to be performed (Windeyer et al., 2014; Chigerwe et al., 2015). As stated in the Materials and Methods, if no cause of death was listed and a calf was treated for BRD within $30 \mathrm{~d}$ of its death date, the calf was categorized as BRD mortality. This may have led to a slightly higher estimated BRD mortality than the true BRD mortality rate due to misclassification bias. It is also important to note that the sample size of 5 dairies may not completely represent California dairies in all management practices areas.

The case fatality rate of $2.37 \%$ for BRD reported in this study was lower than the 7.1, 9.4, 13.8, and $6.0 \%$ BRD case fatality rates reported in previous studies (Sivula et al., 1996; Donovan et al., 1998; Windeyer et al., 2014; Urie et al., 2018). The lower case fatality rate in the current study may be due to differences in calf management between previous and the current study herds. Some potential differences relate to the study being performed in California because having dedicated calf caretakers is a common practice on larger California dairies. Another unique California practice is year-round outdoor housing, which is possible in the temperate California environment but not in most of the United States. Alternatively, the difference could be due to a bias in selection of dairies toward more intensively managed dairies that had good recordkeeping. For the current study, dairies were only considered for participation if they kept detailed records on preweaning calves. If this detail extended to care of calves, it could be that calves treated for BRD on these dairies had a lower risk of dying of BRD because of more effective treatment of BRD by calf caretakers.

Both BRD-caused and all-cause mortality models showed a negative association between administration of modified live vaccines to the dam and calf mortality. We did not identify the specific vaccines used because comparing vaccines was not the study objective. Dubrovsky et al. (2019) showed that calves born to dams vaccinated with a modified live vaccine were $67 \%$ less likely to develop BRD. A previous study explored the association between dam vaccination and calf mortality but it was not found to be significant (Windeyer et al., 2014). Such differences between studies may be due to

Table 3. Final logistic regression mortality models for bovine respiratory disease (BRD) and all-cause mortality in 11,465 calves on 5 dairies in California between March 2015 and July 2016 from birth to weaning ${ }^{1}$

\begin{tabular}{|c|c|c|c|c|c|c|}
\hline \multirow[b]{2}{*}{ Mortality model and variables } & \multirow[b]{2}{*}{ Coefficient } & \multirow[b]{2}{*}{ Odds ratio } & \multirow[b]{2}{*}{$\mathrm{SE}$} & \multicolumn{2}{|c|}{$95 \% \mathrm{CI}$} & \multirow[b]{2}{*}{$P$-value } \\
\hline & & & & Lower & Upper & \\
\hline Summer & Referent & - & - & - & - & - \\
\hline Spring & 1.275 & 3.578 & 1.404 & 1.658 & 7.720 & 0.001 \\
\hline \multicolumn{7}{|l|}{ Maternity bedding changed per month } \\
\hline $0-3$ times & Referent & - & - & - & - & - \\
\hline 4-9 times & -1.318 & 0.268 & 0.094 & 0.135 & 0.531 & $<0.001$ \\
\hline$\geq 9$ times & 1.184 & 3.266 & 1.914 & 1.036 & 10.300 & 0.043 \\
\hline \multicolumn{7}{|l|}{ All-cause mortality model } \\
\hline Modified live vaccine administered to dam & -0.600 & 0.549 & 0.079 & 0.414 & 0.727 & $<0.001$ \\
\hline
\end{tabular}

\footnotetext{
${ }^{1}$ Management practices were surveyed every 2 to 3 mo throughout the study. The variable "dairy" was treated as random effect in the models. ${ }^{2}$ Season was assigned for the date at which the calf was treated for BRD and included the months December to February (winter), September to November (fall), June to August (summer), March to May (spring).

${ }^{3}$ Referent was calf born as a singleton.
} 
the multiple dairies and multiple vaccines used in the current study. Another possibility is that the Windeyer et al. (2014) study had a sample size that was not large enough for statistical significance. The current study had over 10,000 calves (322 died) compared with 2,830 calves (98 died) in Windeyer et al. (2014). The association between vaccination of dams using modified live vaccines and BRD-specific or all-cause mortality should be interpreted with caution because only one of the study dairies changed its vaccination protocol for dams during the study; nevertheless, that dairy contributed $63.5 \%$ of the study calves. Given this sample size, it is probable that vaccination of dams does have a protective effect against BRD-specific and all-cause mortality in preweaned calves through colostrum intake.

An important finding of the current study was the association between season of BRD diagnosis and BRD-caused mortality. Specifically, calves that were diagnosed with BRD in spring had 1.28 times the risk for BRD-caused mortality as calves diagnosed with BRD in summer (the month with the lowest hazard of BRD mortality). Previous research on season and calf mortality in California, performed in 1975, noted the highest rate of mortality in the fall (September-November) and March was the month with the highest mortality; however, that study did not examine BRDcaused mortality (Martin et al., 1975). Martin et al. (1975) hypothesized that months of climatic change with high daytime temperatures and low nighttime temperatures stress the calves' immune systems as they need added energy for thermoregulation; the increase in calves' energy requirements during heat stress leads to energy deficit, poor thermoregulation, and higher mortality. These results suggest that it may be most beneficial for California dairy producers to aggressively control BRD during seasonal changes in climate by implementing preventive management practices in spring and potentially also in fall.

The negative association in the current study between larger volumes of milk fed and all-cause mortality, specifically in calves over $21 \mathrm{~d}$ of age, contrasts with Huber et al. (1984), which reported a correlation between greater amounts of milk fed and higher number of days medicated for female calves; however, the latter study only utilized 16 calves ( 8 female and 8 male) and no correlation was found between increases in milk intake and days medicated in the male calves. As discussed in Drackley (2008), 3.7 L of milk per day may not meet the energy requirements of calves, potentially lowering their immunity. The 2014 NAHMS report stated that $57.7 \%$ of all calf raising operations in the United States fed only 3.8 quarts a day of milk or milk replacer (USDA, 2016). Although this exceeds the recommended volume for maintenance, feeding practice analysis reported in the 2014 NAHMS report found an association between preweaning mortality and the amount of fat in the calves' liquid diet (Urie et al., 2018). Specifically, calves fed $\leq 0.15$ versus $\geq 0.22$ $\mathrm{kg}$ of fat/d in the preweaning liquid diet had 3 times greater odds of mortality (Urie et al., 2018). Although the amount of milk fed and amount of fat in the diet is not a perfectly correlated relationship, they are both measures of the amount of nutrition a calf is receiving and suggest that lack of adequate nutrition may play a major role in increasing the risk of preweaning mortality.

Twins were found to have a 1.7 times greater risk of mortality than singleton calves in the current study. Other studies had similar findings, with Gulliksen et al. (2009) reporting a 1.3 times increased risk of mortality in the first week of life and an overall increased risk of death for calves born as a twin. Mellado et al. (2014) examined the period from birth to $21 \mathrm{~d}$ of age and found a $5 \%$ increase in rate of mortality in twins compared with singleton calves. An increase in mortality risk in twin calves may be due to competition for nutrients during gestation, resulting in reduced vigor and health status after birth. Results of the studies by Gulliksen et al. (2009) and Mellado et al. (2014) suggest that it may be beneficial for calf caretakers to closely monitor calves that are born as twins for any clinical signs of illness during the preweaning period.

In the current study, we identified that frequent changing of the bedding material in the maternity pen reduced the risk of BRD-caused mortality. However, the risk of BRD mortality increased if the bedding was changed more than 9 times per month. The most likely explanation for the decrease in BRD incidence with increase in pen cleanings is the reduction in the potential concentration of pathogens that calves are exposed to during birth and immediately afterward. Both Windeyer et al. (2014) and Sivula et al. (1996) noted maternity pen practices but found no association between maternity pen management and mortality. This may be due to other differences in maternity pen variables such as location of the pens (isolated versus adjacent to milking cow pens), the type of bedding used in the pen, and the frequency of changing the bedding material. It would be beneficial to examine the role of maternity bedding in a study that controls for these other maternity pen variables and controls for maternity bed cleaning frequency at its association with preweaning calf health.

Passive transfer of immunity in the first few hours of life is a critical goal of calf management programs because it is predictive for calf morbidity and mortality (Donovan et al., 1998; Virtala et al., 1999; Beam et al., 2009; Urie et al., 2018). Mahendran et al. (2017) reported $31 \%$ FPT using a cut-off of $5.2 \mathrm{~g} / \mathrm{dL}$, whereas 
another recent study, Johnson et al. (2017), used a slightly different cutoff of $5.0 \mathrm{~g} / \mathrm{dL}$ and found $24.4 \%$ FPT. These 2 rates are both higher than the $16.8 \%$ FPT observed in the current study. Notably, in the current study, bull calves had a higher rate of FPT (28.6 vs. $15.7 \%$ in females) similar to rates found in other recent studies. This may indicate that differences in FPT are related to colostrum management and administration and that the female calves in this study may have had better colostrum administration and overall management than the male calves or calves in the other studies reporting higher rates of FPT. Another study conducted on a single California dairy reported a mean TP of $5.9 \mathrm{~g} / \mathrm{dL}$ in calves fed $4 \mathrm{~L}$ of first-milking pooled colostrum and had 0\% FPT (Williams et al., 2014). Despite the lower rates reported in this study, there is still room for improvement; Chigerwe et al. (2015) suggest that serum TP levels of 5.8 to $6.3 \mathrm{~g} /$ dL are optimal, and Urie et al. (2018) concluded that the industry serum IgG concentration target should be increased to $15 \mathrm{~g} / \mathrm{L}$, cut-off values that would have increased the number of calves deemed to have FPT in this study. Specifically, using a higher cut-off of 5.8 to $6.2 \% \mathrm{~g} / \mathrm{dL}$ for serum TP was found to be associated with an absence of calf mortality in preweaned dairy calves (calves with congenital issues or trauma resulting in death were excluded from this study; Chigerwe et al., 2015). Despite the relatively lower rate of FPT in our study compared with other recent studies, research indicates that there remains room for improvement and that calves in this study above the cut-off of $5.2 \mathrm{~g} / \mathrm{dL}$ may still have been at risk of mortality (Chigerwe et al., 2015).

\section{CONCLUSIONS}

Preweaning mortality rates on California dairies in the current study were slightly lower than those reported in recent studies encompassing most regions of the United States. Rates of preweaning mortality and BRD-specific mortality were associated with management practices, including cleanliness of maternity pens, feeding calves on a higher plane of nutrition, and vaccination of dams. Other factors that may affect the risk of mortality include season of BRD diagnosis and whether a calf was born as a twin or singleton.

\section{ACKNOWLEDGMENTS}

The current study was funded by competitive grant \#1753 "Risk assessment, welfare analysis, and extension education for dairy calf respiratory disease management in California," to Sharif Aly provided by the University of California Division of Agriculture and
Natural Resources and the USDA National Institute of Food and Agriculture (Washington, DC; Animal Health project CALV-AH-360). The authors thank the veterinary practitioners, dairy producers, and calf caretakers for their participation and all the researchers who helped collect the data.

\section{REFERENCES}

Beam, A. L., J. E. Lombard, C. A. Kopral, L. P. Garber, A. L. Winter, J. A. Hicks, and J. L. Schlater. 2009. Prevalence of failure of passive transfer of immunity in newborn heifer calves and associated management practices on US dairy operations. J. Dairy Sci. 92:3973-3980.

Buczinski, S., G. Forte, D. Francoz, and A. M. Belanger. 2014. Comparison of thoracic auscultation, clinical score, and ultrasonography as indicators of bovine respiratory disease in preweaned dairy calves. J. Vet. Intern. Med. 28:234-242.

Calloway, C. D., J. W. Tyler, R. K. Tessman, D. Hostetler, and J. Holle. 2002. Comparison of refractometers and test endpoints in the measurement of serum protein concentration to assess passive transfer status in calves. J. Am. Vet. Med. Assoc. 221:1605-1608.

Chigerwe, M., J. V. Hagey, and S. S. Aly. 2015. Determination of neonatal serum immunoglobulin $\mathrm{G}$ concentrations associated with mortality during the first 4 months of life in dairy heifer calves. J. Dairy Res. 82:400-406.

Donovan, G. A., I. R. Dohoo, D. M. Montgomery, and F. L. Bennett. 1998. Associations between passive immunity and morbidity and mortality in dairy heifers in Florida, USA. Prev. Vet. Med. $34: 31-46$.

Drackley, J. K. 2008. Calf nutrition from birth to breeding. Vet. Clin. North Am. Food Anim. Pract. 24:55-86.

Dubrovsky, S. A., A. L. Van Eenennaam, B. M. Karle, P. V. Rossitto, T. W. Lehenbauer, and S. S. Aly. 2019. Epidemiology of bovine respiratory disease $(\mathrm{BRD})$ in preweaned calves on California dairies: The BRD 10K study. J. Dairy Sci. 102:7306-7319. https://doi .org/10.3168/jds.2018-14774.

Gulliksen, S. M., K. I. Lie, T. Loken, and O. Osteras. 2009. Calf mortality in Norwegian dairy herds. J. Dairy Sci. 92:2782-2795.

Huber, J. T., A. G. Silva, O. F. Campos, and C. M. Mathieu. 1984. Influence of feeding different amounts of milk on performance, health, and absorption capability of baby calves. J. Dairy Sci. 67:2957-2963.

Johnson, K. F., N. Chancellor, C. C. Burn, and D. C. Wathes. 2017. Prospective cohort study to assess rates of contagious disease in pre-weaned UK dairy heifers: Management practices, passive transfer of immunity and associated calf health. Vet. Rec. Open 4:e000226.

Lago, A., S. M. McGuirk, T. B. Bennett, N. B. Cook, and K. V Nordlund. 2006. Calf respiratory disease and pen microenvironments in naturally ventilated calf barns in winter. J. Dairy Sci. 89:4014-4025.

Lora, I., F. Gottardo, B. Contiero, B. Dall Ava, L. Bonfanti, A. Stefani, and A. Barberio. 2018. Association between passive immunity and health status of dairy calves under 30 days of age. Prev. Vet. Med. 152:12-15.

Losinger, W. C., and A. J. Heinrichs. 1997. Management practices associated with high mortality among preweaned dairy heifers. J. Dairy Res. 64:1-11.

Love, W. J., T. Lehenbauer, P. H. Kass, A. L. Van Eenennaam, and S. S. Aly. 2014. Development of a novel clinical scoring systemfor onfarm diagnosis of bovine respiratory disease in pre-weaned dairy calves. PeerJ 2:e238.

Mahendran, S. A., R. Booth, L. Beekhuis, A. Manning, T. Blackmore, A. Vanhoudt, and N. Bell. 2017. Assessing the effects of weekly preweaning health scores on dairy calf mortality and productivity parameters: Cohort study. Vet. Rec. 181:196.

Martin, S. W., C. W. Schwabe, and C. E. Franti. 1975. Dairy calf mortality-rate-Influence of management and housing factors on 
calf mortality-rate in Tulare county, California. Am. J. Vet. Res. 36:1111-1114.

McGuirk, S. M., and S. F. Peek. 2014. Timely diagnosis of dairy calf respiratory disease using a standardized scoring system. Anim. Health Res. Rev. 15:145-147.

Mellado, M., E. Lopez, F. G. Veliz, M. A. De Santiago, U. MaciasCruz, L. Avendano-Reyes, and J. E. Garcia. 2014. Factors associated with neonatal dairy calf mortality in a hot-arid environment. Livest. Sci. 159:149-155.

Schaffer, A. P., R. L. Larson, N. Cernicchiaro, G. A. Hanzlicek, S. J. Bartle, and D. U. Thomson. 2016. The association between calfhood bovine respiratory disease complex and subsequent departure from the herd, milk production, and reproduction in dairy cattle. J. Am. Vet. Med. Assoc. 248:1157-1164.

Shivley, C. B., J. E. Lombard, N. J. Urie, D. M. Haines, R. Sargent, C. A. Kopral, T. J. Earleywine, J. D. Olson, and F. B. Garry. 2018. Preweaned heifer management on US dairy operations: Part II. Factors associated with colostrum quality and passive transfer status of dairy heifer calves. J. Dairy Sci. 101:9185-9198.

Sivula, N. J., T. R. Ames, and W. E. Marsh. 1996. Management practices and risk factors for morbidity and mortality in Minnesota dairy heifer calves. Prev. Vet. Med. 27:173-182.

Tozer, P. R., and A. J. Heinrichs. 2001. What affects the costs of raising replacement dairy heifers: A multiple-component analysis. J. Dairy Sci. 84:1836-1844
Urie, N. J., J. E. Lombard, C. B. Shivley, C. A. Kopral, A. E. Adams, T. J. Earleywine, J. D. Olson, and F. B. Garry. 2018. Preweaned heifer management on US dairy operations: Part V. Factors associated with morbidity and mortality in preweaned dairy heifer calves. J. Dairy Sci. 101:9229-9244.

USDA. 2016. Dairy 2014, Dairy Cattle Management Practices in the United States, 2014. USDA Animal and Plant Health Inspection Service (APHIS): Veterinary Services (VS), Center for Animal Health and Epidemiology (CEAH), Fort Collins, CO.

Virtala, A. M., Y. T. Grohn, G. D. Mechor, and H. N. Erb. 1999. The effect of maternally derived immunoglobulin $\mathrm{G}$ on the risk of respiratory disease in heifers during the first 3 months of life. Prev. Vet. Med. 39:25-37.

Williams, D. R., P. Pithua, A. Garcia, J. Champagne, D. M. Haines, and S. S. Aly. 2014. Effect of three colostrum diets on passive transfer of immunity and preweaning health in calves on a California dairy following colostrum management training. Vet. Med. Int. 2014:698741. https://doi.org/10.1155/2014/698741.

Windeyer, M. C., K. E. Leslie, S. M. Godden, D. C. Hodgins, K. D. Lissemore, and S. J. LeBlanc. 2014. Factors associated with morbidity, mortality, and growth of dairy heifer calves up to 3 months of age. Prev. Vet. Med. 113:231-240. 